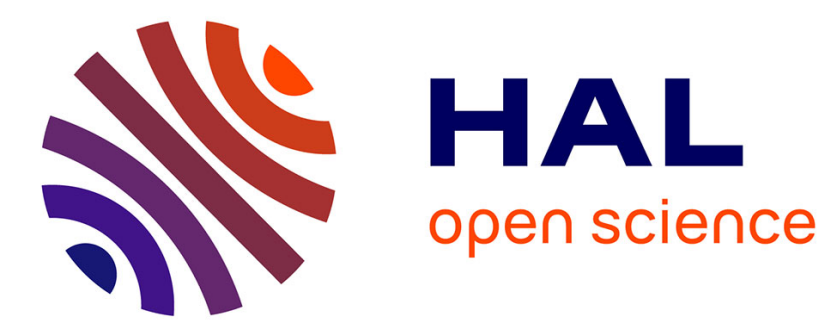

\title{
" La Bulgarie en crise démographique
}

Gérard-François Dumont, Marta Sougareva, Nikolai Tzekov

\section{To cite this version:}

Gérard-François Dumont, Marta Sougareva, Nikolai Tzekov. " La Bulgarie en crise démographique. Population et avenir, 2005, 671, pp.17-19. 10.3917/popav.671.0017 . halshs-00770358

\section{HAL Id: halshs-00770358 \\ https://shs.hal.science/halshs-00770358}

Submitted on 24 Jun 2013

HAL is a multi-disciplinary open access archive for the deposit and dissemination of scientific research documents, whether they are published or not. The documents may come from teaching and research institutions in France or abroad, or from public or private research centers.
L'archive ouverte pluridisciplinaire HAL, est destinée au dépôt et à la diffusion de documents scientifiques de niveau recherche, publiés ou non, émanant des établissements d'enseignement et de recherche français ou étrangers, des laboratoires publics ou privés. 


\section{La Bulgarie en crise démographique}

Les conséquences du soviétisme n'ont pas fini de se faire sentir. L'opinion européenne a pu en prendre conscience en novembre 2004 avec l'Ukraine. La Bulgarie, futur membre de l'Union européenne, avec ses 7,8 millions d'habitants, offre un autre exemple. La fin de la société soviétisée s'y accompagne d'une crise démographique qu'illustrent des chiffres saisissants.

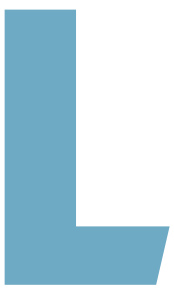

e tournant s'effectue le 10 no vembre 1989 , avec la chute du pouvoir de Todor Jivkov, à la tête du Parti communiste depuis 1954, soit pendant 35 ans $^{1}$ ! Auparavant, la Bulgarie peut être considérée comme le plus soviétisé des pays de l'empire, ainsi que l'attestent par exemple les attitudes v oulant « faire table rase du passé » vis-à-vis de cer taines minorités.

\section{Une émigration provoquée}

L'histoire démographique contemporaine est en effet marquée par une politique, appelée par la pr opagande officielle " le procès de renaissance ", et concernant l'attitude du pouvoir vis-à-vis des cito yens bulgares ayant des origines ethniques turques, ou de confession musulmane. Elle s'explique en partie par la mémoire de cinq siècles d'occupation ottomane (du XV e au XIXe siècle), qui n'ont véritablement pris fin qu'en 1908, 30 ans après le premier traité de Libération, et qui entretient l'idée d'une Turquie plutôt menaçante et, paradoxalement, désirée pendant la période communiste, car elle représentait ces pays étrangers où il était interdit de se rendre. Depuis les années 1970, le gouv ernement conduit une politique de changement for cé des noms ar abes en noms bulgares : cette politique est d'abor d dirigée contre les bulgares mahométans (personnes d'origine ethnique bulgar e ayant adopté la religion musulmane), puis, à partir de 1984, contre les Bulgares turcs. Vers 1985, cette politique est déployée plus intensément pour « bulgariser » le pays. Elle pr ovoque des révoltes, des conflits ethniques, et fait des victimes. Elle pr ovoque une émigration d'environ 320000 bulgares turcs vers la Turquie voisine, dont à peine 100000 sont $r$ entrés dans leurs lieux de naissance depuis la période de dév eloppement démocratique des années 1990. L es conséquences démographiques de cette vague migratoire, à laquelle s'ajoute l'émigration rurale, sont particulièrement visibles, surtout à l'échelon local. Un gr and nombre de communes, sur tout des communes rurales, au Nord-Est et au Sud-Est, ont perdu 20 à $50 \%$ de leur population. Ainsi des régions peuplées de bulgares turcs, qui avaient une croissance naturelle positive, se sont-elles transformées en noyaux de dépopulation.

\section{La fécondité stimulée, puis effondrée}

La démographie contemporaine de la Bulgarie peut être divisée en deux périodes distinctes : av ant la période de démocratisation (dont le début se situe à la fin de 1989), et depuis. Pendant la première période, l'économie du pays est or ganisée d'une manièr e totalitaire : les entr eprises sont la propriété de l'Etat, qui est le seul patron. Il n'y a pas de chômage apparent, tout le monde est obligé de travailler, à l'exception des étudiants, des personnes malades ou handicapés, et des mèr es ayant des enfants de moins de 3 ans et, bien entendu, des retraités.

À la fin des années 1960, une politique pro-nataliste est mise en place. Elle comprend d'une part des incitations positives (surtout économiques : avantages pour les jeunes mariés quant à l'accès aux logements et aux pr estations des banques, primes à la naissance d'un enfant, pr estations familiales ${ }^{2}$ ), d'autre part des mesur es restrictives (par exemple, les personnes non mariées, les familles sans enfants, et les familles qui n'ont qu'un seul enfant payent un impôt dit « sur le célibat »). L'accès libre à l'avortement, institué en 1958, est remplacé entre 1968 et 1973 par un régime restrictif, notamment pour les femmes mariées ayant moins de 2 enfants. Dans ce contexte, la fécondité et la nuptialité se maintiennent élevées jusqu’à la fin des années 1980.

Puis la démocratisation, accompagnée d'une ouverture à l'information et aux styles de vie de l'Occident, a pour effet une importante diminution de la fécondité et de la nuptialité. À la question : «Quels ont été les principaux facteurs de cette baisse? ", il n'est pas facile de répondre. Le nombre des naissances est d'abord fonction du nombre

\section{LA FÉCONDITÉE EN BULGARIE}

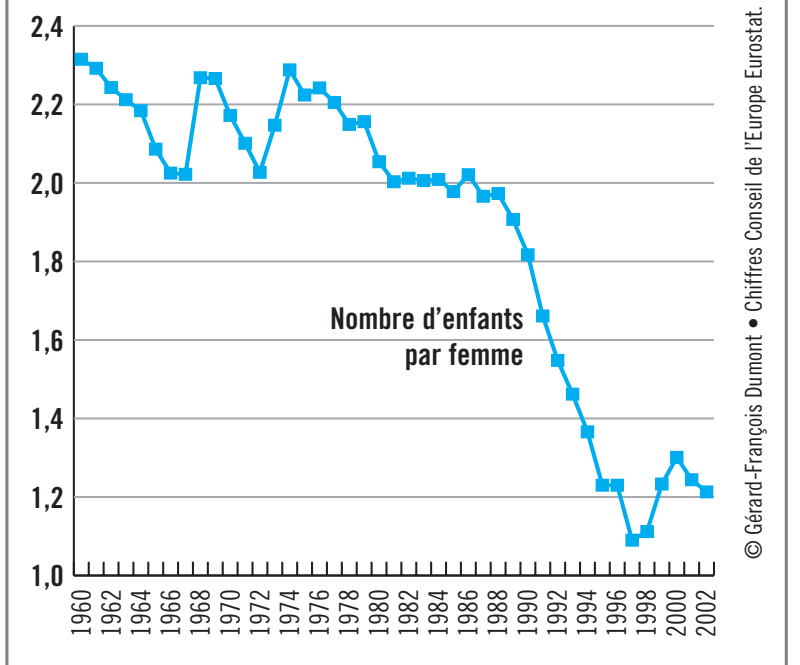

par Gérard-

François

DUMONT

Marta

SOUGAREVA*

et Nikolai

TZEKOV*

Directeur de recherche, Centre d'études sur la population, Académie des sciences de la Bulgarie.

Chercheur, Centre d'études sur la population, Académie des sciences de la Bulgarie.

Cet événement se produit lors d'une réunion du comité central du parti communiste, qui, ensuite, après un vote de ses membres, change son nom pour se dénommer " Parti socialiste bulgare »PDB. Une deuxième force politique, I'Union des forces démocratiques UFD, se constitue, d'abord en tant que coalition, puis en tant que parti. Le modèle politique "bipolaire» est complété par plusieurs autres partis, dont le « Mouvement pour les droits et les libertés » MDL, formé sur un principe ethnique, nommée encore " le parti turc », et le Parti agrarien.

2 Néanmoins, ce qui peut paraître curieux, la politique pro-nataliste introduisait des aides dont le montant augmentait progressivement (en fonction du rang de l'enfant) jusqu'au troisième enfant, tandis que, pour le quatrième et les enfants de rangs plus élevés, ce montant était limité. 
des femmes en âge fécond ; or , ce nombre a diminué à la suite de l'émigration, dont les données sont mal connues. Deuxièmement, les naissances arriv aient auparavant assez tôt (l'âge moyen des femmes à la première naissance était inférieur à 22 ans au début des années 2000). Elles se sont déplacées aux âges plus av ancés, et, dans les années 2000, les pr emières naissances arrivent à un âge supérieur de 1,5 ans en mo yenne par rapport à 1989.

\section{Le mariage et les naissances retardées}

Avant 1989, comme les premières naissances, les mariages intervenaient donc tôt, en compar aison avec les autr es pays de l'Europe, entre 21 et 22 ans en moyenne. Ce modèle de mariage précoce et quasi-universel est conforme à la ligne de Hajnal concernant la démogr aphie européenne.

Depuis 1993, l'âge moyen au premier mariage des femmes célibataires a entrepris une forte progression.

\section{L'ÂGE MOYEN AU PREMIER MARIAGE ET À LA PREMIÈRE NAISSANCE}

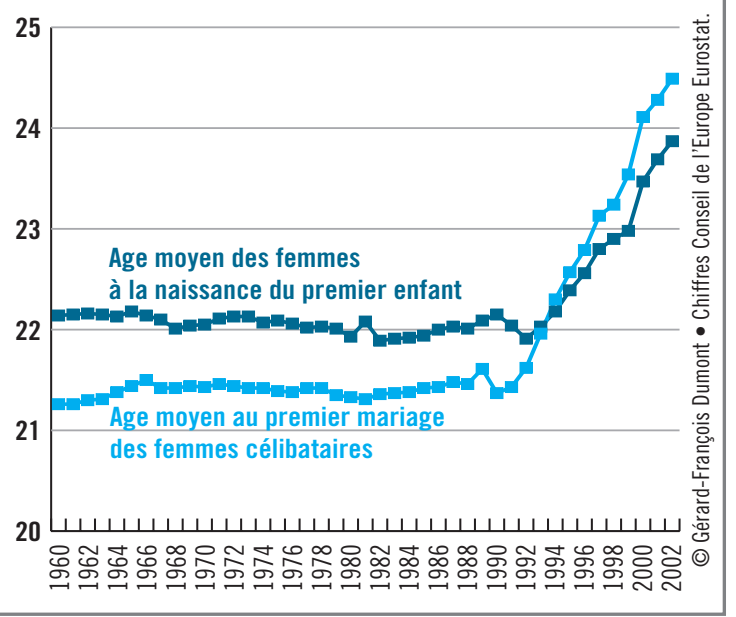

\section{Des avortements encore nombreux}

Un autre trait caractérisant la période communiste était le recours extrêmement fréquent à l'avortement en tant que moyen de planification familiale. Des années 1970 aux années 1990, le nombre des avortements est supérieur à celui des naissances, soit plus de mille av ortements pour mille naissances.

Le niveau élevé de l'av ortement est alors typique pour tous les pays de l'Est. Toutefois, parmi les pays de l'Est, la Bulgarie se trouve dans le groupe des pays à très haut taux d'avortement, se rapprochant des niv eaux les plus élevés jamais observés dans le monde, surtout en prenant en considération la sous-estimation du nombre des avortements par les statistiques officielles.

La politique à l'égar d de l'av ortement a tr ansformé les attitudes et les valeurs de la population, en diminuant la gravité de l'av ortement dans la conscience des gens, et ses effets négatifs sur la santé physique et mor ale. Cette politique coïncidait et correspondait avec une période de délégitimisation de l'église et de la $r$ eligion. La pratique
3. LES NAISSANCES ET LES AVORTEMENTS

\section{EN BULGARIE}

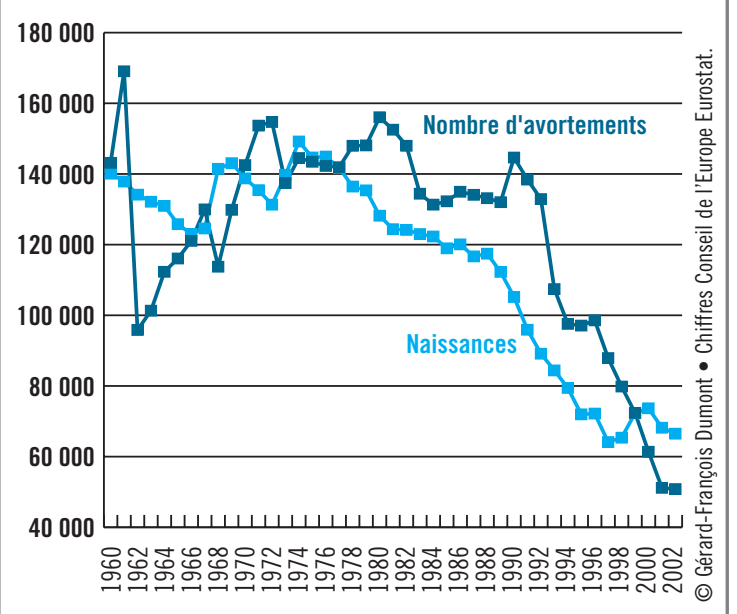

religieuse était presque totalement supprimée, à l'exception de quelques lieux privés, et les pratiquants risquaient des difficultés diverses dans leur vie et leur carrière.

Les données officielles sur l'avortement (qui sous-estiment les chiffres réels, sur tout après 1990, la v aleur de cette sous-estimation restant inconnue) mettent en évidence une baisse depuis les années 1990.

\section{Le divorce culpabilisé sous les soviets}

En 1985, un nouveau code de famille est introduit, dans le dessein de diminuer les divorces. Les personnes divorcées sont soumises à des sanctions diverses, y compris des problèmes dans leur travail et leur carrière professionnelle. Par exemple, une personne divorcée ne peut pas occuper un poste dirigeant dans le parti communiste. Cette législation, qui culpabilise le divorce, est contraire à celle des pays occidentaux. Son but est une fécondité élevée, car la "famille socialiste stable » est considérée comme favorable à la naissance d'un plus grand nombre d'enfants.

Elle entraîne effectivement une baisse brutale des divorces en 1985. Ensuite, à partir de 1995, le nombre des divorces reste autour de 10000

\section{LES DIVORCES EN BULGARIE}

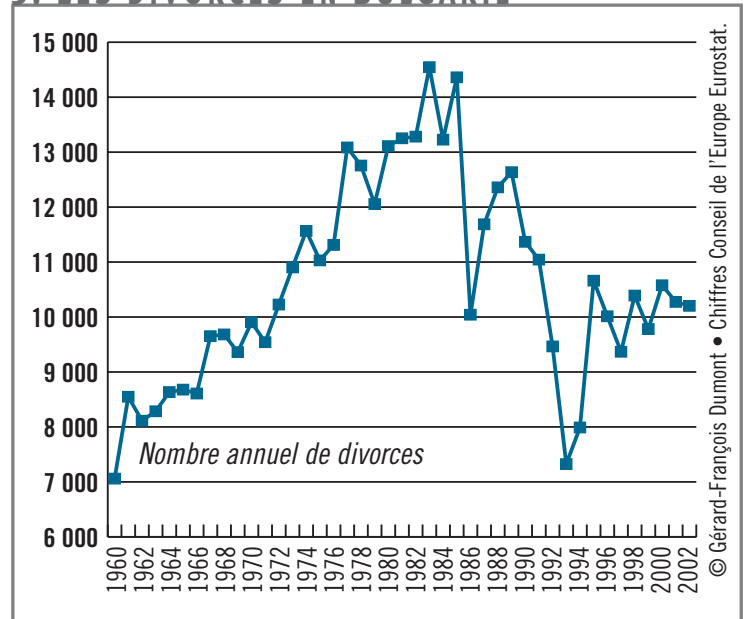




\section{La répartition ethnique}

La composition ethnique de la population est complexe, car la définition de certains groupes n'est pas uniforme selon les diverses sources d'information. Sur les 7,8 millions d'habitants, les principaux groupes ethniques sont : Bulgare, Turc (estimé à 760000 personnes), et R oms (environ 370 000) ; le premier est lar gement majoritaire, et les deux derniers varient selon les régions du pays, et selon les modes d'enregistrement. Les Turcs vivent surtout dans le nord-est du pays et les musulmans bulgar ophones, appelées Pomaks, dans le Sud.

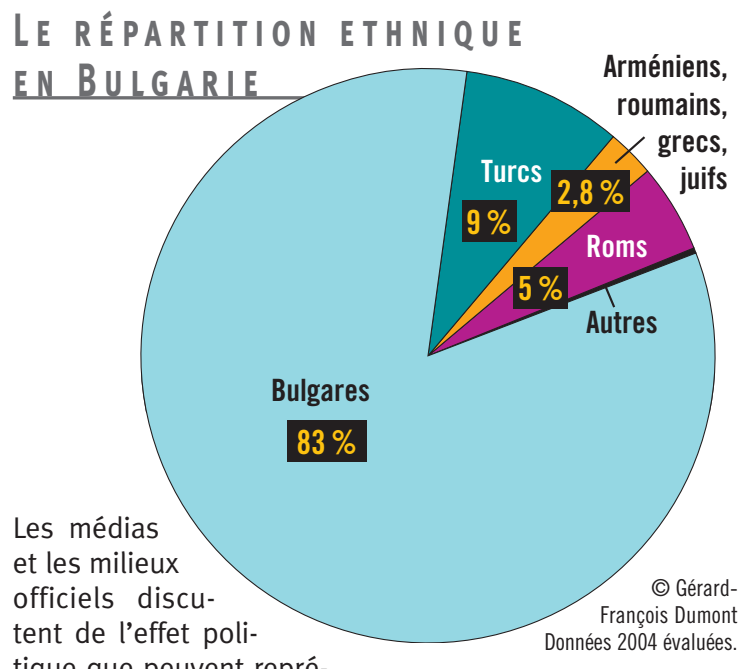

tique que peuvent repré-

senter les minorités ethniques, et surtout les Roms, ayant une fécondité nettement plus élevée que le reste de la population. On constate une hausse progressive du pourcentage de la population des ethnies non bulgares. Les recherches montrent un fort pourcentage de nouveau-nés roms. Mais les chiffres qui circulent sont discutables.

En outre, plus importants sont les problèmes économiques, la grande pauvreté et le chômage que connaissent la plupart des Roms, et aussi le fait qu'une grande partie de leurs enfants ne fréquentent guère l'école. La plupart des enfants roms ne terminent pas l'école secondaire, et leur présence dans les universités est pratiquement nulle. Le manque d'éducation et d'information aggrave la situation de cette population, et augmente le conflit potentiel existant entr e cette minorité et la majorité de la population. En plus, les mariages 3 dans le groupe Roms se font très tôt (à l'âge de 14-15 ans pour les filles et de 17-18 ans pour les garçons), et, presque aussitôt, les couples commencent à avoir des enfants. Donc la procréation est accélérée, malgré la diminution récente du nombre moyen d'enfants par couple. À la base d'observations fragmentaires, on peut estimer que le nombre moyen d'enfants par femme est environ 4-6 pour les générations Roms se trouvant à la fin de leur carrière reproductive, et que les plus jeunes se limiteront à 2-4 enfants par femme en moyenne.

\section{Vieillissement et dépopulation}

Un des sérieux pr oblèmes qui se pose désormais concerne le dépeuplement de cer taines régions bulgares. Des villages et des petites villes perdent de la population, et leur structure par âge se détériore à cause de l'émigration et d'un taux net de reproduction inférieur à l'unité. Les régions qui souffrent le plus de dépopulation sont celles du Nord-Ouest du pays (qui est principalement agraire), et toutes les communes proches des frontières nationales.

Dans nombre de villages, la structure par âge est très vieillie, et le taux de masculinité très bas, le pour centage des femmes étant supérieur à celui des hommes. De vastes surfaces agricoles, autr efois travaillées, restent à l'abandon, le paysage devient parfois sauv age et même désertique. Le dépeuplement entr aîne des difficultés aussi dans les services sociaux, les transports, la gestion et l'organisation de l'infrastructure, la vie culturelle, la vie religieuse, et même en matièr e de sécurité personnelle (hausse des délits). Les fermetures d'écoles et la suppression de lignes d'autobus incitent une émigr ation encore plus intense de population active et qualifiée.

De façon génér ale, la société bulgar e des années 2000 ressent un sentiment général de désorientation (en raison des changements $r$ adicaux du système économique et politique), qui se mêle parfois à une nostalgie du passé, exprimée par la phrase : "Nous étions bien mieux sous Todor Jivkov ». Cette nostalgie est typique surtout chez les couches les plus pauvr es (ex. les R oms, les personnes âgées vivant isolement, les chômeurs, etc.)

La situation démogr aphique en Bulgarie se car actérise donc par une véritable crise. Le modèle de comportement démographique du passé, corr espondant à une situation d'économie dirigée, et une politique pro-nataliste prononcée, s'est modifié en raison de la libéralisation des normes sociales en matière de famille, comportement sexuel, etc. À ces difficultés s'ajoutent les pr oblèmes de la recherche en démographie, qui, après la période communiste, ouvertement soumise aux intérêts politiques et idéologiques du parti communiste (et de l'Etat), $n$ 'a guère progressé pendant ces dernières années. Il en résulte souvent des interprétations incorr ectes des données existantes et des spéculations politiques.
${ }^{3}$ Mais il ne s'agit pas forcément de mariages légaux. Les mariages chez les Roms sont souvent conclus d'après leurs propres rites, et sans être officiellement enregistrés. La fécondité hors mariage que l'on observe en Bulgarie (42\% en 2003) est largement le résultat de la structure ethnique des naissances, et s'explique notamment par celles de femmes roms. Ceci est une observation non officielle, puisque les registres des naissances (comme ceux des décès) ne contiennent pas d'information sur le groupe ethnique.

D'après les données du dernier recensement (2001), la proportion des Roms aux âges de procréation est environ $10 \%$, et dans certaines régions, bien plus élevée. Les femmes dans ce groupe commencent à avoir des enfants à un âge très jeune, et elles ont une descendance finale plus importante que les autres groupes.

\section{LA DENSITÉ DE LA BULGARIE}

